\title{
A Participatory Perspective on Cross-Cultural Design
}

\author{
Kasper Rodil ${ }^{1}$ \\ Dep. of Architecture, Design and Media Technology \\ Aalborg University \\ Sofiendalsvej 11, 9200 Aalborg SV \\ Denmark \\ kr@create.aau.dk
}

\begin{abstract}
Designers face a number of challenges in terms of how to design interactive systems with indigenous groups. Every layer of development faces obstacles from designing localized interfaces to facilitating prototype evaluations in the wild. This article argues for the importance of continuous user involvement and participatory design. This is highlighted through explaining ongoing research in the creation of a 3D visualization knowledge management system to support preservation of indigenous knowledge (IK) in Africa. Through the sharing of experiences from the field I underpin the importance of acknowledging users' expertise and knowledge about the design context. Through presentation of a selection of these challenges in localizing systems development I wish to raise awareness of an required sensitivity to cultural differences in IT.
\end{abstract}

Keywords: indigenous knowledge, visualization, interactive system, participatory design, user-centered design, field study, Namibia

\section{Introduction}

Being users and designers in Western countries we design towards a wide array of available technologies. Through institutionalized teaching of design and acquired principles derived from a plethora of empirical user studies and projects developers can usually rely on these guidelines when creating new projects. Since most of our interface design relies on principles firmly rooted within the demographic from which designers and users share similar characteristics. Large parts of this demographic have been part of the development of interfaces and HCI for a relatively long time. E.g. a user can often apply previously acquired IT skills from one OS to the next generation of that OS etc. Besides having established conventions of how to design interfaces, the users and designers also often share similar underlying conceptual structures -while applied design guidelines in turn sustain the conceptual structures of the users/designers.

One might argue that when users and developers have similar 'expectations' for an end-product the necessity for radical design seems small. By following design trends we also communicate with users in an expected manner, which naturally 
has a great value for the user who can interact with a familiarly looking interface. The designs originating from the same contextual background as the users might not seem radical to users from that context, but for users from other nationalities/cultures a proposed system can pose too much friction to be of use. When designing for indigenous groups or users with a different cultural background to the domain of Western principles of design, development immediately faces barriers on fundamental areas of interaction design, interface design and diversity in ontological categorization of objects. As Heukelman (2006) states: "Working with a user interface designed for 1st world users could make inexperienced computer users feel that it was not intended for their use." [1]. The author further states: "Designers often erroneously believe that they know what the users need, especially inexperienced computer users." [1].

Tools in the Western designer's toolbox are established and well assimilated by Western users, but by perceiving the tools as universal/global solutions the possibility of ignoring localization as a cardinal point for understanding the users and the context is plausible. Early in the process of a cross-cultural project the designer must consider if he/she believes that the design components such as UI icons(is the concept of icons even universal?) are globally transferable or not, and whether this distinction is important for the design or not. While Bidwell \& Winschiers-Theophilus (2010) support the claim of the importance of localization: "Localizing interaction design in Africa is critical for improving usability and user experience for African populations" [2]. Winschiers-Theophilus (2009) further explains: "Looking at the history of cross-cultural IT design and usability evaluation shows the originally naïve assumption that IT, being value neutral, only needs to be slightly adapted to its new environment" [3].

The objective of this article is not to question Western design traditions, but to highlight their possible inadequacy when introduced to a completely unfamiliar domain. Both the Western and the unfamiliar domain are by comparison full of differences and unique opportunities. Thus when jumping between those domains designers are subjected to re-evaluate their concepts, which in return might create new ideas and knowledge. One of the ambitions for the following pages is to convey a message that bridging the gap between fundamentally different users/co-designers and designers is not a slight adaptation in design thinking. Nor should the designer be a sovereign authority but could engage in participatory design activities to challenge his own concepts, and seek inclusion of other concepts than those originating from his own context.

The ambition for the first part of the chapter is to create 'food-for-thought' for developers and designers wishing to embark into cross-cultural systems design by outlining some differences/challenges in understanding the users in unfamiliar contexts. Following is an introduction to Participatory Design (PD), then motivation and an overview of a project focusing on developing a locally managed indigenous knowledge system for elders in a Herero village in Namibia is described. The chapter ends with some chosen examples (and references to other publications with more detail) about the importance of PD. The intention for 
the chapter is not to provide a system description but to highlight some challenges in cross-cultural understanding of and inclusion of differences into the design process.

\section{A Critical Perspective on Approaches to Understand Users}

When developing a system together with people from another culture, there are naturally obvious differences and similarities between those parties. Some of these differences and similarities are meaningful to include in the design solutions, since they represent local values and sometimes represent an uniqueness not readily perceived by external designers. While IT systems in general are Western constructs based on Western epistemologies, a growing need and realization of cultural differences rendering the notion of universal design flawed has led to approaches to understand the 'other'. According to Hofstede's (2006) research on 'culture dimensions' the majority of the designers in the project team described here has contrasting origin (Scandinavia) compared to the Namibian context on several societal structures and personal value beliefs [4]. The Namibian context and Western designer's origin differs according to Hofstede in the following areas [4]:

- Feminity vs. Masculinity:

"Minimum emotional and social role differentiation between the genders." Vs. "Maximum emotional and social role differentiation between the genders."

- Individualism vs. Collectivism:

"Speaking one's mind is healthy" and "Personal opinion expected: one person one vote" Vs. "Harmony should always be maintained" and "Opinions and votes predetermined by in-group."

- Power Distance:

"Older people are neither respected nor feared." Vs. "Older people are both respected and feared."

Hofstede's theory on cultural dimensions represents one perspective on understanding core differences between cultures and its people. The cultural dimensions do not provide guidelines for developing perfect systems nor should they be used as generalizable facts for a plethora of diverse cultures, languages, and religions in Africa. It provides an outline of the differences between co-designers and designers, but not a detailed guideline for understanding the context.

As Irani et al. (2010) explain: "Some have sought to predict and understand these problems of translating HCI knowledge by drawing on taxonomic models of culture where members of cultural groups are characterized by traits and averages." [5].

Kamppuri (2012) further questions the generalizable nature of these models: "In the same way, even though cultural dimensions are based on questions related to a particular area of life, such as work, it is assumed that the differences found 
between countries are similar in other contexts, too." [6]. Namibia is an example of clear differences in the national population and is inhabited by 13 different tribes who along with other ethnic groups constitute a highly diversified user base not fit for generalizations. While some of these groups might have similarities with a foreign designer, an adaptation to this group can not rely on intuition alone. As stated by Teasley et al.: "'Professional intuition' is neither a sufficient nor reliable methodological foundation for producing an 'appealing perceptual experience' in interactive computer systems. "[7]. Irani et. al explain: "ICT4D designers face challenges transporting both design conventions and processes of HCI across cultures. HCI's visual conventions have proven not to be universal systems effective in the US may fail utterly in Japan or South Africa." [5].

The elaborate article on basic psychological and perceptual differences in cultures presented by Henrich et al. (2010) describe the assumption that the generalization of WEIRD (Western Educated Industrialized Rich and Democratic) users/subjects cannot be justified on a global scale [8]. The main conclusion is that the WEIRD population is "particularly unusual compared with the rest of the species" and "there are no obvious a priori grounds for claiming that a particular behavioral phenomenon is universal based on sampling from a single subpopulation" [8]. "The sample of contemporary Western undergraduates that so overwhelms our database is not just an extraordinarily restricted sample of humanity; it is frequently a distinct outlier vis-a-vis other global samples. It may represent the worst population on which to base our understanding of Homo sapiens" [8]. Relating to Henrich's research where samples from one population might inform design in another population leads to the questioning of attempts to provide global guidelines in order to localize cross-cultural design.

By using Bennett's terminology, the designer can choose to hold onto "denial of difference" or seek "integration of difference" [9]. I must make clear here that not all cross-cultural projects require similar nuancing or levels of localization. The project described later requires IK preservation in the 'design' of the system and as 'content' in the system, but I believe the consideration makes sense no matter the project scope. Namibia is a post-colonial country thus a mix of religions, tribes and world views. Being freed from apartheid in the 1990's, and now a young democracy(by Western measurements). Does Namibia have a singular culture or does it constitute of various cultures? Developers should ask (probably at least) three fundamental questions.

- What constitutes the origin and empirical foundation for the knowledge leading to decisions in the design phase?

- What level of localization is needed?

- Is there in the design process room for local differences to be manifested?

By perceiving people living within the same national borders as being belonging to the same group and pertaining the same world views there is a great risk of suppressing the perspectives of the minorities. I would always argue for a much more nuanced view on people than by pooling them into overall user groups. Does it make sense to perceive descendants of European colonialists and 
Hereros to be having similar values and world views? It might be that the experiences we share and knowledges being brought forward in one Herero community is different from the next Herero community etc. Even within the same community I would claim we could find different opinions towards many things. Cultural models are one way of looking at differences, another approach could be by being informed through in situ dialogue and inclusion of personal perspectives. I must underpin that these two could support each other in our aspirations to understand users and the context. Where the perspective from one elder in one Herero village probably is not the perspective of everyone, nor is the generalized values enough for localizing IT design.

\section{An Introduction to Participatory Design}

This section presents a brief background for PD as a methodology for users involved in the design process of interactive systems.

User-Centered Design (UCD) as a discipline is spanning a plethora of methods and techniques to facilitate input from end-users to help shape or solve a design problem. Through methods such as interactive paper prototyping [10], personas, use cases and visual ethnography etc. the ambition is to make sure the design fits the users and their contexts. UCD houses an array of iterative conceptual models to ensure user involvement and usability of a system. One of the most known models for evolutionary acquisition of user input is the spiral model [11], which conceptually and practically directs when users through iterations are involved. UCD have proven valuable in contexts/domains where design negotiations are founded on shared principles of power relations, gender roles, and cultural norms through shared means of communication. PD is a process-oriented variant of UCD. One of the tenets of PD is an emphasis of the continuous involvement of users through empowering and egalitarian principles. As Sanders et al. (2010) explain: "Participatory Design today is an emerging design practice that involves different non-designers in various co-design activities throughout the design process." [12].

PD is historically rooted in Scandinavian socio-technical developments for workplaces and within trade unions in the 1970s and 1980s to democratically "rebalance the power of workers and management" [13]. The onset was to explore and apply methods to implement interactive systems that would fit workers and their skills. Nygaard and associates were pioneering PD with the NJMF project initiated in Norway in 1970 [14]. Later followed the DEMOS project in Sweden in 1975 [15]. Project DUE, Denmark 1978 [16]. The UTOPIA project started in 1981 as collaboration between The Nordic Graphic Workers' Union and researchers from Denmark and Sweden [17]. One of the points from the project is that design professionals having technological skills should understand the worker context. The worker (or user) often lack the technological understanding, but have knowledge on context and have the skills within that context. 
As Dearden \& Rizvi based on [13] explain in their elaborate review: "A common theme has been one of 'mutual learning' where technology designers learn about the setting where technology is to be used, and users continuously learn about technology design and designers" [18]. Kensing and Blomberg (1998) explain: "the epistemological stand of PD is that these types of knowledge are developed most effectively through active cooperation between workers (and increasingly other organizational members) and designers within specific design projects." [13]. Other projects related to the Scandinavian PD tradition are reported by Ehn (1993) in [17].

Although PD has a political origin in workplace democracy the methods associated have increasingly been used to bring users and developers closer. As reported by Gregory (2003), PD is also considering discussions of values in design and that conflicts and contradictions are to be seen as resources in design [19].

As Muller states regarding the political background of PD: "Many researchers and practitioners in PD (but not all) are motivated in part by a belief in the value of democracy to civic, educational, and commercial settings a value that can be seen in the strengthening of dis-empowered groups (including workers), in the improvement of internal processes, and in the combination of diverse knowledge to make better services and products." [20]. The participatory designer should be a facilitator of communication, a gatekeeper responsible for the fusion of differing knowledges and emphasizing polyvocality as a unique opportunity to create 'better products'.

A cardinal point in $\mathrm{PD}$ is regarding the decision making and power balances between designers and participants. Users in UCD processes are urged to provide feedback and ideas during their involvement. They represent a voice in the decisions on terms, interaction design, functionality etc. but the design professionals responsible for a given system solely decide if they decide to listen. As Bratteteig and Wagner (2012) explain it: "While in commercial design projects there may be some sharing of power, participatory design (PD) opens up for systematically including users and other stakeholders in the decision processes in design. It is assumed that their knowledges and skills are also valid in the exploration and evaluation of both big and small decisions." [21].

While Scandinavian PD backbone has a political motive to empower users and an ambition to include domain knowledge into the design of systems with objectives of for instance mutual learning, our design approach is of a more conceptual nature. We are not participatory designers from a particular political nerve but from a realization that our attempts to make 'good systems' would be fruitless unless designed together. Our aspirations to empower the local community is to infuse their perspectives and world views, which is essential since an objective is to represent these local view points within the interface and system design.

The ambition for the following pages is to explain some of the reasons for perceiving the co-designers in Namibia as essential partners in producing functional localized design. 


\section{Overview of a Project in Designing Localized Interactive Systems to Support Preservation of Indigenous Knowledge}

\subsection{Background}

Namibian Herero youths are currently assimilating knowledge from a curriculum influenced by characteristics of a modern society while being contextually de-situated from decades of traditional transfer of IK, cultural values and sustainable living in the rural areas of Namibia. The modus of traditional dissemination in the rural areas has for generations been through informal masterapprenticeship by practical learning and as oral knowledge transfer between youths and knowledgeable elders in the rural areas. Besides the obvious local benefits in acquiring knowledge on husbandry, herbal lore etc. the tacit knowledge transferred through inter-personal interaction with and within the context effectively adds to preserving local culture, customs and traditions.

Wenger (1998) articulates the often tacit and uncodable knowledge situated within a community of practice as a 'repertoire' [22]. Being absent from the rural areas for large parts of the year the youths are unable to exercise this repertoire locally, thus exacerbating the process of local knowledge management. Confronted with a knowledge paradox on what type of knowledge would aid them and the eco-system in the rural areas in their respective future most optimally, the youths are per governmental regulations left without a choice. Being situated in remote schools for large parts of the year, the effect is a disruption of interpersonal knowledge transfer between curators (the elders) and future curators (youths).

In Namibia, a majority of urban migrants return to their villages in the rural areas, regularly on short visits and permanently after many years of living in the cities. They return to an unmanaged and unmaintained knowledge system. UNESCO highlights the importance of preservation of IK: "The UNESCO Convention for the Safeguarding of the Intangible Cultural Heritage highlights the importance of developing tools and measures to preserve and transmit cultural knowledge of indigenous peoples in terms of traditions, practices, expressions, knowledge and skills that are created and shaped by communities in close interaction with their environment" [23].

\subsection{Project Overview}

In order to preserve and transmit IK between Namibian community groups separated by age and location, our research project aims to develop an IK management system, which villagers (especially elders) can utilize to manage IK unassisted. The IK content comprises of elders having recorded multimedia, primarily video recordings on where to find healing herbs or how to slaughter a goat according to customs etc., but a system for the elders to store these clips in is much needed.

A major concern and design challenge is the fundamental difference between 
the orientations of African cultures and the orientations of Western cultures governing designers and technology. It is important to investigate under what conditions the corpus of IK can be preserved and mediated and represented for city living youths with a minimal loss of IK content and meaning. Being developers primarily from other value systems and cultural orientations we are aware of the fact that we are not able to decide, select and handle which particular knowledge is important/relevant. Our endeavors into creating an IK system, investigating the clash of cultures in HCI and the use, reshaping and creation of new methodologies in PD have proven fruitful. But as every layer have been investigated new layers keep surfacing making us rethink and re-evaluate our approaches.

Through introductions made by local researchers in Namibia we have since 2009 worked closely with a community of the Herero tribe located in the Kalahari Desert. The tribe has traditionally been pastoralists in the rural areas of Namibia since settling down (17th and 18th century) in the region. Our approaches to help the community preserve their cultural heritage have been approved by village elders, whom traditionally have decision power in the community and custodians of the IK. Until now the research has been directed to investigating and prototyping possible approaches in creating a system for preserving IK, which is co-designed through methods of PD and where all phases in the development are negotiated locally. Fig.1 shows the evaluation of a prototype running on a 10.1 inch Motorola Xoom tablet.

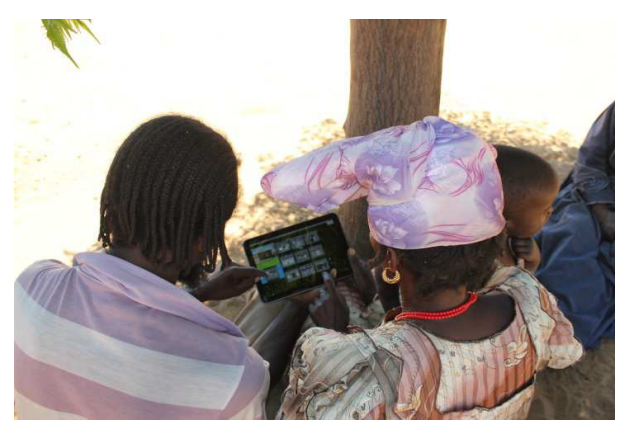

Fig. 1. Dialogue on a prototype.

Initial work in the project has shown the inadequacy of text-based interfaces to facilitate knowledge management for the so called digitally illiterate elders. Based on ethno-graphical field observations and reflections a number of design options, including speech output, picture-based input and tangible prototypes have been explored, as described by Kapuire \& Blake [24]. These advances have all expanded our understanding of the domain we are engaged in. Most importantly they have from the beginning of the project gradually involved the elders, thus also added to the building of trust and helped clarifying our intentions. 
While attitudes towards the Western academic scene and nature of researchers is usually good in the Western world, academics are not always attributed objectivity and honesty when seen from an indigenous perspective. For instance as noted by Braun et al. (2013), indigenous societies are well-accustomed to researchers flying in, grabbing what they need, advancing their careers and then leaving again [25]. The elders have sovereign authority on allowing and supporting our involvement in their village, and in granting us permission to document our trips with video recorders and cameras.

We conduct the design in-situ using several design probes as means for polyvocal dialogue, and consider the village elders as co-designers. Due to the nature of PD and our attachment to the research site's people everything becomes intertwined in activities as research and as not-research. Due to the project's setting and the nature of PD it is not easy (nor always desired) to pick out individuals for a usability test etc. I would argue for that if our system does not function in the community environment with a burning sun and people unfamiliar with IT, we are not producing anything successful besides our own advancement. Thus instead of the dominating researcher wishing to assume control of the experiment, we often let the interaction unfold naturally while observing.

The Hereros are as a majority of indigenous groups valorizing collectivism, then why should the research methods reflect Western use of IT and individualism? After all, measuring number of clicks in the interface or measuring speed of interaction might not be relevant since some cultures do not rush into action before careful consideration. We are accustomed to the thought of IT as being almost value-free, but IT can be a different experience for non-Western users. As noted by Oyugi et al.(2008) designers should "...consider the degree of replication of Western approaches to usability methods.." [26]. A study by Vatrapu \& Prez-Quiñones (2006) show how interview/interviewee relations can affect the analysis and results: "When the usability methods involve human-human interaction, such as is the case with structured-interviews; then the interaction of the cultures of the two participants must be considered." [27].

Where usability can investigate errors or pinpoint weaknesses in the interaction with the prototype, it is usually defined by the visiting researcher. Similarly are interviews usually following the researcher's agenda. While these approaches can be efficient in a design process, they have inborn difficulties challenging the concepts the researcher design with.

Dialogue is as already emphasized important for understanding and investigating the prototypes' suitability. Dialogue as a participatory activity for challenging one's concepts is vital for representing local view points within the interface and system design. Lakoff \& Johnson provide a way to look at it [28]:

- Ideas(or meanings) are objects.

- Linguistic expressions are containers.

- Communication is sending.

This practically means that when the designer evaluates i.e. an interface icon he should be cautious not to measure only the success or failure of his idea, but to challenge the concept of an icon, since it might not exist locally. Due to dialogue 
being a linguistic form, the researcher should be careful not to run his argument as a 'battle', thus he would loose the cooperative aspects of dialogue, which is important to further his understanding of the concept of the icon.

\subsection{D Visualizations as a Design Approach}

Since 2010, we have investigated the potential of 3D visualizations as supportive visual meta-data in sense of creating a virtual context for IK content recorded as multimedia content by village elders, and investigating how $3 \mathrm{D}$ worlds can mediate the knowledge transfer between youths and elders [29]. The context embodying the videos can holistically provide visual information on individuals, the nature, objects etc. not easily perceived or missing from the video recordings, thus widening and adding to the information stored in the IK videos.

An example of a virtual scenario could be the collection of herbs for a bad stomach. The video recording could display an interview with an elder elaborating on location and how to find the specific herb in the bush. The reconstructed $3 \mathrm{D}$ scenario would visually depict the surrounding environment such as time of the day, people involved in the collection etc. Besides adding to the information from the collected videos, the scenarios are a different approach to manage the structuring and assigning of 'visual' meta-data, which must rely on local concepts and is highly culturally dependent.

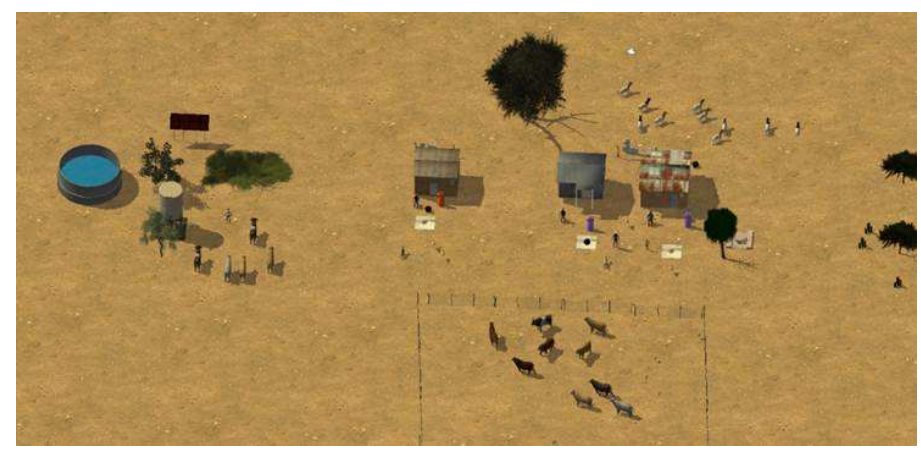

Fig. 2. The figure shows the core idea of a virtual world, where 3D objects constitute the 'building blocks for the recorded IK.

Some of the current points of focus are investigating any cultural difference of shape, color, camera perspectives [30] of the virtual models for the system. These areas are foundational for in the next phases to have tools for the co-designers to create custom scenarios free from our interference. We have introduced drawing as a method to be informed on conceptual and visual representation of objects and argue "...that this helps to democratize the design and that these methods 
can be used in the broader contexts of bridging the gap of understanding across cultures where technology or language may fall short [31].

Thus hopefully being representations with closer fitting to the co-designers perspective, ambitions and world-views.

We are currently exploring how elders can design these scenarios themselves; as described in [32], but a future system could potentially house a large corpus of in-situ collected video material. Thus the requirement for a repository and a smooth integration of user generated content is needed. A functional idea in the Western world could be a sorting of videos based on textual meta-data in a large database, where users upload their videos and provide tags for later retrieval by others based on those categorizations and keywords. The work of Hughes and Dallwitz (2007) stress the shortcomings of traditional databases when trying to sort IK. They describe that physical objects may be culturally restricted to be either for both genders or purely for one gender to see [33]. Thus the representations as multimedia of real objects directed the authors to create three parallel databases. Currently we have a focus on the possible inter-cultural differences between ontologies and taxonomies as underlying structures for such databases and interface structures (for an example see Rodil et al. (2013)) [34].

With the visualization approach we deliberately bypass any requirements for skills in textual literacy due to previous experiences in the village. From a PD point of view we argue that text interfaces dis-empower our co-designers. In this approach it must be envisioned that the user is visually browsing what could resemble a virtual village and then e.g. decides to see how a traditional goat slaughter is conducted according to customs. While results are promising with this attempt we are much aware that the complexity of the system might not be possible to align with a pure visualization approach.

The specifications for the final system are ambiguous at this point. Thus being in the middle of the process Brereton et. al (2011) describe as: "The challenge for any design project is to build relations and cross-cultural understanding, so as to ensure that local aspirations are articulated effectively into socio-technical design outcomes." [35].

If we already know the end result we would implicitly disregard user input and stray from a participatory process. Our approach is an oscillation between 'going forward' with a prototype. Then if being promising for co-design and accepted by the parties involved we backtrack to research why it has been effective. Naturally the prototypes reflect thoughtful considerations from developer side. But as experienced many times in the field and reported by other researchers good intentions and sound design choices do not necessarily provide successful prototypes when evaluated locally.

\section{Participatory Design In Cross-Cultural IT Design}

The majority of our design team does not have an ethnic origin in the context were we design artifacts. As stated by Marcus (2006): "It is difficult for design- 
ers/analysts to escape being biased culturally. All designed artifacts are cultural objects." [36]. There is arguebly some truth to Marcus' statement, but probably the 'cultural' part comes from the designer. As Suchman (2002) states: "our vision of the world is a vision from somewhere that it is inextricably based in an embodied, and therefore a partial perspective which makes us personally responsible for it." [37].

The difficulties of exporting systems design across cultures have been reported many times and surely we have experienced it ourselves. From the beginning we have come to terms with the cultural differences as problematic for the design of the IK system and have used PD as a way to consider people engaged with technology and how designs could be harmonized with local influence. In our project having a clear objective of preserving and transferring local IK, we can consider the external designers as potential filters or facilitators in this transfer. We accept that the 'cultural objects' being prototyped might interfere with the representation of culture in this transfer. There is a risk of transforming the knowledge when we represent it through a to the context unfamiliar artifact. And if the knowledge is captured by externals, then synthesized and represented by those externals, it would be questionable to claim that no distortion is taking place?// Mutema (2003) explains on the interpretation of IK: "Understanding is made possible through dialogue, conversation and communication between the researcher and the actors. The inter-subjective nature of the research process allows for the researcher's interpretations to be checked, reinterpreted and evaluated by the actors." [38].

In order to ensure that the system is aligned with the local perspective, we have since the beginning actively sought to involve local future users as being co-designers who are critical towards the ideas presented. The critical nature towards ideas put forward is a tenet of PD, but difficult to facilitate. In this case the notion of power relations can aid in explaining it. For instance it is not new to receive critique from students and test participants in the Western world, if the researcher asks for it. In cultures with different values in power distance, it is more complicated, since it might not be polite to critique people perceived to be higher in the hierarchy. Thus we have invested significantly more effort in explaining that we seek critique.

Iivari (2004) describes three levels of user involvement as being either a 'consultative', 'representative' or 'consensus' type [39]. Iivari describes consensus as: "the responsibility of design is assigned to users, who are continually involved in the design process and have power to make decisions" [39]. We perceive the village elders as being consensual co-designers empowered to shape the IK system under their terms and requirements. The other community members in the village are in traditional sense to be considered as users primarily involved in evaluating the usability of prototypes. Empowerment of users into being co-designers is a central theme in PD.

Ertner et. al (2010) explain on the empowerment of users: "In order for the 
designer to empower the users, user participation requires more than simply uttering wishes and participate sporadically in the process users need to gain actual power in decision making and direct influence in the entire process in order to be empowered." [40]. Designers in traditional PD are seen as being able to empower users and thus improving the design. In our particular project, I would claim that the elders equally empower us (along with our technological skills) to partake in designing the IK system.

\subsection{Mutual Learning}

One's skills are essential for any successful design, but that skills should be traded or reflected not only to empower users/designers in decision making, but because the trained designer and indigenous co-designers possess different strengths that must be shared, critiqued and evaluated in dialogue. The dialogues are important for enriching the 'contextually illiterate' designer with an updated skill set and in bringing awareness of different perspectives important for developing in a crosscultural domain. And for the co-designer to acquire technological knowledge -thus being in a more balanced position to critique whatever the designer brings forward. Pearson \& Robinson (2013) have a good point when they stress that how can people criticize something if they do not know what they want and the technological capabilities being available [41]. Although PD has mutual learning as a guiding principle it can also come with a cost to design systems intended for a marginalized demographic.

During the development the co-designers gradually familiarize themselves with new technologies, which move them closer to the trained developers in terms of IT skills. While this might be important for the dialogue about prototypes, it is in the meantime important that some anchor remain in the end-users' demographic. Recently we have focused on the design export to other sites to ensure that the designs produced with the elders remain successful within the broad community group. Years of familiarization with technology and design might affect the whole project, since the co-designers might be so skilled and proactive in a system's short-comings, that basic concepts are learned, but not transferable to the rest of the co-designers' demographic.

\section{Experiences With Prototypes}

From several field trips to our pilot community discussing prototypes and their use, we have learned that fundamental areas must be considered before conducting field studies. One of the primaries is the balancing act of appropriate hardware technology behind a software prototype. Findings presented in [32] highlight the radical shift in co-designer involvement and critique when we exchanged laptop and mouse driven software prototypes with tablets and touch input functionality. When presenting the first proof-of-concept of a $3 \mathrm{D}$ visualization to the elders, we experienced reluctance in interacting with the laptop 
and mouse. The co-designers sat in a larger group commenting on what they saw on the screen, but did not actually try to interact with the prototype. As quoted from [32]: "He (the elder) said that on the other prototypes (laptops) they had to use a mouse, which was difficult, this (tablet) is easy cause he just has to use his hand" (in situ translation). The concept proved to create much less friction when technology device and actual system were both more intuitive to the elders.But from a PD perspective the technology was an active gatekeeper for participation. Designers should consider if their technology of choice hinders participation.

We experienced new perspectives on technology devices and HCI, when these different prototypes were in the hands of the elders. This can be illustrated by findings from a field study investigating how common (common in the Western world) touch input gestures such as two-finger rotation, one-finger drag etc. were perceived in the community and how to decide on implementation of gestures for missing functionality in the prototype called the Homestead Creator. We deliberately left the functionality for rotating virtual objects up for discussion with the community for two reasons. 1) How would they actually try to rotate a virtual object without instructions given? 2) If we informed them of a rotation gesture it would be difficult to decode the local intuitiveness of an implemented choice. And if a local gesture was preferred it is the participatory designer's onus to facilitate inclusion in the methods in contrast to only evaluate the designer's choice.

Another important finding in sense of the involvement of co-designers has been presenting them with proof-of-concepts as rather high fidelity software prototypes, which act as center points of dialogue. Our experience is that when the presented prototype fidelity is too far from an actual designer envisioned output -it becomes harder to critique. That lo-fi can be too far from the vision designers have, thus transferring an abstract concept to people with little experience in IT is difficult. When we presented a smaller part (virtual area of the village); but looking close to the vision of the system of the larger envisioned 3D visualization, we immediately received valuable feedback in sense of ways to recreate the $3 \mathrm{D}$ so that it matches the local views on i.e. virtual cows, trees etc. So far all of our prototypes have elements which could function in a final system, but the important discipline for us is to be ready to change them or discard them. Sometimes we experience something unexpected and have to take several steps back to investigate i.e. how perception of virtual camera perspectives in a virtual world might be different for the community members than Westerners trained in using maps. And common to all the prototypes we have developed together is that none of them are meant as final products per se. They are meant as abstracting down and articulating approaches which in the future scope could be the digital IK management system. Being are aware that we are creating the proofs-of-concept and a valid question could be articulated regarding who the actual designer is. 
That is why we oscillate between various prototypes and methods to inform our (all stakeholders') design.

I believe that there are many technological approaches to the problem, but we have from the beginning decided to defocus on a specific technology and while the co-designers become gradually more aware of the technological possibilities for a system we in the meantime narrow the field of hardware candidates. E.g. in the case of exchanging laptops with tablets we saw a radical shift in ease of use and constraints set by the physical environment, hence we would never in the future settle on using that particular hardware platform for a situated community IK system. One of the lessons we learned from this example is the differing nature of the feedback we received with different combinations of hardware and software prototype fidelity.

\section{Conclusion}

When relating the arguments carried in the article to the context of PD in Namibia, we can raise some ideological 'pillars' to support cross-cultural system's development. The system's design must be designed in such a way that it infers the world views from the context's inhabitants. It must be validated locally due to psychological, perceptual, societal etc. differences.

It has been evident through many field trips to the community that we as designers and system developers can not solely rely on skills previously acquired when designing for a much different context than our own. It has been clear that it is possible to develop prototypes and design human-computer interaction that is better aligned with the elders' and rest of the community's ways of using; to them, new technology. But that all phases of development rely on a premise of localization through Participatory Design, and being critical towards the transferability of findings and design ideas from contexts that are unaligned with the new design space. But even more importantly that the designer questions the universality of his ideas. After all when designing together with people with other perspectives it is necessary to include their concepts, thus also actively challenging your own concepts.

\section{Acknowledgements}

I wish to thank all Erindiroukambe's community members for friendships and for giving me personal experiences I treasure highly. I also wish to thank the members of the project team for their dedication of investigating these important challenges, and especially my supervisors for continuous dedication and feedback.

\section{References}

1. Heukelman, D.: Can a user centered approach to designing a user interface for rural communities be successful? In: Proceedings of conference CHI-SA. (January 2006) 51-58 
2. Bidwell, N.J., Winschiers-Theophilus, H.: UNDER DEVELOPMENT: Beyond the Benjamins: toward an African interaction design. interactions 17(1) (January 2010) 32-35

3. Winschiers-Theophilus, H.: The Art of Cross-Cultural Design for Usability. In Stephanidis, C., ed.: Universal Access in Human-Computer Interaction. Addressing Diversity. Volume 5614 of Lecture Notes in Computer Science. Springer Berlin / Heidelberg (2009) 665-671

4. Hofstede, G.: Dimensionalizing Cultures: The Hofstede Model in Context. Online Readings in Psychology and Culture (2006)

5. Irani, L., Vertesi, J., Dourish, P., Philip, K., Grinter, R.E.: Postcolonial computing: a lens on design and development. In: Proceedings of the 28th international conference on Human factors in computing systems. CHI '10, New York, NY, USA, ACM (2010) 1311-1320

6. Kamppuri, M.: Because deep down, we are not the same: values in cross-cultural design. interactions 19(2) (March 2012) 65-68

7. Teasley, B., Leventhal, L., Blumenthal, B., Instone, K., Stone, D.: Cultural diversity in user interface design: are intuitions enough? SIGCHI Bull. 26 (January 1994) 36-40

8. Henrich, J., Heine, S.J., Norenzayan, A.: The weirdest people in the world. Behavioral and Brain Sciences 33(2-3) (2010) 61-83

9. Bennett, M.J.: A developmental approach to training for intercultural sensitivity. International Journal of Intercultural Relations 10(2) (1986) 179-196

10. Buxton, B.: Sketching User Experiences: Getting the Design Right and the Right Design. Morgan Kaufmann Publishers Inc., San Francisco, CA, USA (2007)

11. Boehm, B.W.: A spiral model of software development and enhancement. Computer 21(5) (May 1988) 61-72

12. Sanders, E.B.N., Brandt, E., Binder, T.: A framework for organizing the tools and techniques of participatory design. In: Proceedings of the 11th Biennial Participatory Design Conference. PDC '10, New York, NY, USA, ACM (2010) 195-198

13. Kensing, F., Blomberg, J.: Participatory Design: Issues and Concerns. Comput. Supported Coop. Work 7(3-4) (January 1998) 167-185

14. Nygaard, K.: The iron and metal project: trade union participation. In Sandberg, A., ed.: Computers Dividing Man and Work Recent Scandinavian Research on Planning and Computers from a Trade Union Perspective. Number 13. Swedish Center for Working Life, Demos Project Report no.13, Utbildningsproduktion, Malmø, Sweden. (1979) 94-107

15. Ehn, P., Sanberg, A.: Management Control and Wage Earner Power (Foretagsstyrning och Lontagarmakt). Falkoping: Prisma (1979)

16. Kyng, M., Mathiassen, L.: Systems development and trade union activities. Computer Science Department, Aarhus University (1979)

17. Ehn, P.: Scandinavian design: On participation and skill. In Schuler, D., Namioka, A., eds.: Participatory Design. Erlbaum (1993) 41-77

18. Dearden, A., Rizvi, H.: Participatory IT design and participatory development: a comparative review. In: Proceedings of the Tenth Anniversary Conference on Participatory Design 2008. PDC '08, Indianapolis, IN, USA, Indiana University (2008) 81-91

19. Gregory, J.: Scandinavian approaches to participatory design. International Journal of Engineering Education 19(1) (2003) 62-74

20. Muller, M.J.: The human-computer interaction handbook. L. Erlbaum Associates Inc., Hillsdale, NJ, USA (2003) 1051-1068 
21. Bratteteig, T., Wagner, I.: Disentangling power and decision-making in participatory design. In: Proceedings of the 12th Participatory Design Conference: Research Papers - Volume 1. PDC '12, New York, NY, USA, ACM (2012) 41-50

22. Wenger, E.: Communities of practice: Learning, meaning, and identity. Cambridge University Press, Cambridge (1998)

23. Unesco: The UNESCO Convention for the Safeguarding of the Intangible Cultural Heritage [online]http://www.unesco.org/culture/ich/en/convention/ [accessed March 12 2013].

24. Kapuire, G.K., Blake, E.: An Attempt to Re-Organise Digital Indigenous Knowledge Representations to Merge Local and Technological Paradigms. In: IKTC 2011. (2011) 72-78 [proceedings available]http://www.indiknowtech.org [accesed March 12 2013].

25. Braun, K.L., Browne, C.V., Ka'opua, L.S., Kim, B.J., Mokuau, N.: Research on Indigenous Elders: From Positivistic to Decolonizing Methodologies. The Gerontologist (July 2013)

26. Oyugi, C., Dunckley, L., Smith, A.: Evaluation methods and cultural differences: studies across three continents. In: Proceedings of the 5th Nordic conference on Human-computer interaction: building bridges. NordiCHI '08, New York, NY, USA, ACM (2008) 318-325

27. Vatrapu, R., Pérez-qui nones, M.A.: Culture and usability evaluation: The effects of culture in structured interviews. Journal of Usability Studies 1 (2006) 156-170

28. Lakoff, G., Johnson, M.: Metaphors We Live By. University of Chicago Press, Chicago (1980)

29. Rodil, K., Winschiers-Theophilus, H., Bidwell, N., Eskildsen, S., Rehm, M., Kapuire, G.: A New Visualization Approach to Re-Contextualize Indigenous Knowledge in Rural Africa Human-Computer Interaction INTERACT 2011. Volume 6947 of Lecture Notes in Computer Science. Springer Berlin / Heidelberg, Berlin, Heidelberg (2011) 297-314

30. Jensen, K.L., Theophilus, H.W., Rodil, K., Goagoses, N.W., Kapuire, G.K., Kamukuenjandje, R.: Putting it in perspective: designing a 3D visualization to contextualize indigenous knowledge in rural Namibia. In: Proceedings of the Designing Interactive Systems Conference. DIS '12, New York, NY, USA, ACM (2012) 196199

31. Winschiers-Goagoses, N., Winschiers-Theophilus, H., Rodil, K., Kapuire, G.K., Jensen, K.: Design Democratization with Communities: Drawing Toward Locally Meaningful Design. International Journal of Sociotechnology and Knowledge Development (IJSKD) 4(4) (2012) 32-43

32. Rodil, K., Winschiers-Theophilus, H., Jensen, K.L., Rehm, M.: Homestead creator: a tool for indigenous designers. In: Proceedings of the 7th Nordic Conference on Human-Computer Interaction: Making Sense Through Design. NordiCHI '12, New York, NY, USA, ACM (2012) 627-630

33. Hughes, M., Dallwitz, J.: Ara Irititja: Towards Culturally Appropriate IT Best Practice in Remote Indigenous Australia. In Dyson, L., Hendriks, M., Grant, S., eds.: Information Technology and Indigenous People. Hershey, PA: Information Science Publishing (July 2007) 146-158

34. Rodil, K., Rehm, M., Winschiers-Theophilus, H.: Homestead Creator: Using Card Sorting in Search for Culture-Aware Categorizations of Interface Objects. In Kotzé, P., Marsden, G., Lindgaard, G., Wesson, J., Winckler, M., eds.: Human-Computer Interaction INTERACT 2013. Volume 8117 of Lecture Notes in Computer Science. Springer Berlin Heidelberg (2013) 437-444+ 
35. Brereton, M., Roe, P., Hong, A.L.: A Cross-Cultural Approach Towards Designing Digital Noticeboards with a Remote Aboriginal Community. In: Indigenous Knowledge Technology Conference 2011. (November 2011) 137-139

36. Marcus, A.: 4. In: Cross-Cultural User-Experience Design. Volume 4045 of Diagrams'06. Springer Berlin Heidelberg, Berlin, Heidelberg (2006) 16-24

37. Suchman, L.: Located accountabilities in technology production. Scandinavian journal of information systems 14(2) (2002)

38. Mutema, G.: Phenomenology, hermeneutics and the study of indigenous knowledge systems. Indilinga: African journal of indigenous knowledge systems 2(1) (2003)

39. Iivari, N.: Enculturation of user involvement in software development organizations - an interpretive case study in the product development context. In: Proceedings of the third Nordic conference on Human-computer interaction. NordiCHI '04, New York, NY, USA, ACM (2004) 287-296

40. Ertner, M., Kragelund, A.M., Malmborg, L.: Five enunciations of empowerment in participatory design. In: Proceedings of the 11th Biennial Participatory Design Conference. PDC '10, New York, NY, USA, ACM (2010) 191-194

41. Pearson, J., Robinson, S.: Developing our world views. interactions 20(2) (March 2013) 68-71 